\title{
Participant views on involvement in a trial of social recovery cognitive-behavioural therapy
}

\author{
Caitlin Notley, Rose Christopher, Joanne Hodgekins, Rory Byrne, Paul French and David Fowler
}

\section{Background}

The PRODIGY trial (Prevention of long term social disability amongst young people with emerging psychological difficulties, ISRCTN47998710) is a pilot trial of social recovery cognitive-behavioural therapy (SRCBT).

\section{Aims}

The PRODIGY qualitative substudy aimed to (a) explore individual experiences of participating in the pilot randomised, controlled trial (recruitment, randomisation, assessment) and initial views of therapy, and (b) to explore perceived benefits of taking part in research $v$. ethical concerns and potential risks.

\section{Method}

Qualitative investigation using semi-structured interviews with thematic analysis.

\section{Results}

Analysis revealed participant experiences around the key themes of acceptability, disclosure, practicalities, altruism and engagement.

\section{Conclusions}

Participants in both trial arms perceived themselves as gaining benefits from being involved in the study, above and beyond the intervention. This has implications for the design of future research and services for this client group, highlighting the importance of being flexible and an individualised approach as key engagement tools.

\section{Declaration of interest}

None.

opportunities to engage young people in new activities linked to their goals and values. The PRODIGY trial (Prevention of long term social disability amongst young people with emerging psychological difficulties, ISRCTN47998710, UKCRN trial registration number: 13341) has recruited young people with high levels of social withdrawal and emerging mental health difficulties. With this qualitative substudy, we specifically sought to address potential ethical considerations of the trial, by asking participants about their experiences of undertaking structured (baseline) assessments (i.e. with regard to burden, potential intrusiveness, etc), and the impact of being randomised to receive a psychological intervention $v$. TAU within a particularly hard to engage, complex and socially withdrawn participant group. We sought to answer the research question 'What are the patient reported experiences of recruitment and initial participation in the PRODIGY trial?' As the SRCBT pilot study might inform future research, we were keen to learn whether the methods used were acceptable to this client group. We therefore felt that qualitative methods were the most appropriate to answer our research question.

\section{Method} team's previous experience that therapeutic aspects of an 'active monitoring' control condition of a randomised trial (supportive listening, access to crisis care, signposting) may offer significant benefits and be highly valued by participants (for example Morrison et al, ${ }^{5}$ Byrne \& Morrison ${ }^{6}$ ).

The qualitative study reported here has been undertaken alongside an ongoing pilot trial of social recovery cognitivebehavioural therapy (SRCBT). SRCBT is a new intervention targeting social disability in young people with emerging psychological difficulties. ${ }^{7}$ Particular emphasis is given to understanding an individual's goals and values in relation to what they want to achieve in the future and instilling hope that this is possible. Barriers to engagement in activity are explored and formulated using a cognitive-behavioural therapy (CBT) approach. The intervention has a strong behavioural focus and individuals are encouraged to test out their beliefs about increasing activity. A further key element is liaising with external agencies to find
This study comprised a qualitative exploratory substudy as part of an ongoing pilot randomised controlled trial (RCT) of SRCBT. Following ethical approval from the Norfolk Research Ethics Committee, a convenience sample of 13 participants was drawn. Participants were recruited over a 6-week period (5 April to 13 June 2013). The sampling approach recruited from all participants who had completed baseline assessments and been randomised, and who had consented to being contacted about the qualitative interviews and being audio recorded. Consenting participants were subsequently approached initially by telephone by a research assistant to arrange a convenient time and place for the interview. The aim was to recruit 12 participants, with good representation across both study sites (Norwich and Manchester) and both trial groups (intervention and TAU). Diagnostic and clinical information about current difficulties and functioning was 
collected routinely from all participants involved in the trial using the Structured Clinical Interview for DSM-IV (SCID), ${ }^{8}$ the Comprehensive Assessment for At-Risk Mental States $(\text { CAARMS })^{9}$ and the Time Use Survey (TUS). ${ }^{10}$ This information is included in Table 1 to contextualise the sample recruited into the current study.

Having obtained informed consent, face-to-face qualitative semi-structured interviews were undertaken (by R.C. and a study research assistant, Becky McIntyre) either in clients' own homes or at an alternative mutually agreed location (for example, general practice surgery). Interviews were undertaken between 1 and 3 months post trial randomisation in order to minimise recall bias of initial trial procedures. Flexible interview guides focused briefly on the history of psychological difficulties, and then on experiences of previous and current treatment. The main focus of the interview guided participants to discuss experiences of being involved in the research study to date, and specifically probed around relevant research procedures, including pre-study information, randomisation, undertaking baseline assessments and experiences to date of the intervention (for intervention group participants only) or of current treatment (for the control group only). All interviews were audio recorded and transcribed verbatim. Interviewers kept a research diary to reflect on the interview and record their thoughts, feelings and reactions to the interview encounter. These notes were referred to during the analysis process.

An inductive thematic approach to analysis was undertaken following data collection. ${ }^{11,12}$ We took a critical realist epistemological stance to data analysis, ${ }^{13}$ recognising that as researchers we assume, although cannot 'know', a fixed reality, but we can understand participant experiences of reality through close examination of their individual perspectives, thus recognising socially determined perspectives of reality. Analysis therefore involved interviewer reflections on interviews, close listening of all interviews, and line by line thematic coding of all transcripts. Coding drew on the words, reported experiences and perspectives of the participants, thus taking an inductive approach without applying any pre-existing analytical framework. R.C. undertook coding with independent coding of four interview transcripts by C.N. and R.B., providing triangulation of analysis and verification. Coding was discussed at regular meetings attended by R.C., C.N., R.B. and J.H. Agreement levels were high when comparisons of independent coding were undertaken. No formal quantitative measure of consensus was applied, as the research team used different words to code the data. Alternative wording was discussed until consensus was reached for the final coding structure. Service user feedback was also sought via the PRODIGY advice team, a panel of young people with experience of using mental health services, to assist with analysis verification. Thematic coding was grouped into key emergent themes, which were summarised under one over-arching theme.

\section{Results}

Thirteen face-to-face qualitative interviews were completed and lasted between 30 and $60 \mathrm{~min}$ (seven Manchester, six Norwich). Six participants were from the therapy arm, and seven from the TAU arm. Only one participant approached did not wish to participate in a qualitative interview, because they preferred not to be audio recorded. Demographic and clinical information about participants is shown in Table 1 .

Exactly 100 participants were recruited into the PRODIGY pilot trial from which the qualitative sample were drawn. Of these, $40 \%$ met at-risk mental state criteria on the CAARMS interview. ${ }^{9}$ Of the trial sample, $51 \%$ were male, and all of the sample were aged 25 years or under, with $69 \%$ falling into the 16-19 years age bracket. Social disability was defined as less than $30 \mathrm{~h}$ per week spent in structured activity. However, the majority of the sample (74\%) were engaged in less than $15 \mathrm{~h}$ of structured activity per week, defined as 'very low' functioning. Based on these sample characteristics, the qualitative subsample reported on in this paper

\begin{tabular}{|c|c|c|c|c|c|c|c|c|}
\hline $\begin{array}{l}\text { Participant } \\
\text { number }\end{array}$ & Age & Gender & Group & Site & SCID research diagnosis & $\begin{array}{l}\text { At-risk } \\
\text { mental } \\
\text { state }^{a}\end{array}$ & $\begin{array}{l}\text { Social } \\
\text { functioning }\end{array}$ & Past treatment ${ }^{\mathrm{c}}$ \\
\hline M001 & 23 & Male & Intervention & Manchester & Generalised anxiety disorder & No & Very low & Early detection service \\
\hline M004 & 18 & Male & TAU & Manchester & None & No & Very low & School counselling service \\
\hline M005 & 23 & Female & TAU & Manchester & $\begin{array}{l}\text { Generalised anxiety disorder, } \\
\text { anorexia nervosa }\end{array}$ & No & Very low & $\begin{array}{l}\text { GP and on waiting list for eating } \\
\text { disorders service }\end{array}$ \\
\hline M006 & 19 & Male & Intervention & Manchester & Depression, social phobia & No & Very low & University counselling service \\
\hline M008 & 20 & Female & TAU & Manchester & Social phobia, agoraphobia & Yes & Low & Short-term therapy \\
\hline M009 & 17 & Male & Intervention & Manchester & Depression, social phobia & Yes & Very low & None \\
\hline M010 & 21 & Female & TAU & Manchester & Depression, social phobia & No & Very low & $\begin{array}{l}\text { University counselling service } \\
\text { and GP }\end{array}$ \\
\hline N002 & 16 & Female & Intervention & Norwich & Specific phobia & Yes & Very low & GP and CAMHS \\
\hline N003 & 18 & Male & Intervention & Norwich & $\begin{array}{l}\text { Social phobia, body } \\
\text { dysmorphic disorder }\end{array}$ & No & Very low & Third sector counselling service \\
\hline N006 & 18 & Male & TAU & Norwich & $\begin{array}{l}\text { Social phobia, generalised } \\
\text { anxiety disorder }\end{array}$ & Yes & Very low & $\begin{array}{l}\text { CAMHS and third sector } \\
\text { counselling service }\end{array}$ \\
\hline N007 & 20 & Male & TAU & Norwich & Depression, social phobia & No & LOW & Youth mental health service \\
\hline N008 & 19 & Male & TAU & Norwich & $\begin{array}{l}\text { Generalised anxiety disorder, } \\
\text { social phobia }\end{array}$ & No & Very low & GP \\
\hline N010 & 21 & Male & Intervention & Norwich & None & No & Very low & $\begin{array}{l}\text { Counselling from a variety } \\
\text { of services }\end{array}$ \\
\hline
\end{tabular}


can be seen as broadly representative of the larger sample, although males were slightly oversampled as a consequence of the conveniences sampling technique ( $9 / 13$ qualitative study participants were male). In the qualitative subsample, 4/13 (30\%) met at-risk mental state, 7/13 (54\%) were 16-19 years, and $11 / 13$ of the qualitative sample were defined as having 'very low' functioning.

Thematic analysis revealed five key themes relating to participant experiences of participating in the trial, which were further grouped under the overarching theme of 'Engagement in psychological research'.

\section{Practicalities}

There was a very strong sense throughout the interviews that it was essential for participants that researchers take a practical and measured approach to research procedures. Twelve of the participants reported that they appreciated flexibility in research appointments, in time, location and also in being able to split the assessments in sessions that suited them in terms of length of time and content.

'When I did my meeting and didn't fill it, there was a paper that I hadn't done, and (researcher) phoned me and asked me if I wanted to come and do it another day or he'd send it, and he sent it, and I brought it to him the next time.' (M008, female)

Others spoke of the practical arrangements of research and an appreciation of flexibility in the sense that it showed empathy on the part of the researchers, and an awareness of the particular difficulties of participants involved in this study.

'I think the fact that they, you know, all of you are willing to come here, just is really appreciated, sort of shows that your aware that some of us can't leave.' (M010, female)

'I thought it was really, really well done, um, she would phone me or text me either the day before, or on the day just to like make sure that I was still up for it, which was really good, and then she would, um, she'd meet me here every time.' (NOO2, female)

There was an appreciation of the skill and training of researchers, who took a person-centred approach. Twelve participants reported that they liked the 'individualised' approach of the researchers.

I like how you can arrange like yourself where you want, the appointments, cos I got (researcher) to come to my house for one of the appointments, and he had a word with my parents, so I thought that was quite good how he could do that.' (NO03, male)

Although comments about the flexibility of the assessments were positive, three participants identified that they would have liked more information about the length of the assessments.

'If I had to suggest anything it might be um explain how long it might take at the start cos like I said nobody told me it so I went thinking it would be another half hour long session but you know if it was told to me it would probably be three hour long sessions then I would have made time at the start.' (N007, male)

\section{Acceptance}

Participants were extremely open and accepting of research procedures and measures. This may be a reflection of the current limited availability of services and intervention for young people as a particular group, but appeared to arise from two main reasons; a thorough understanding of the rationale for the processes or measures used, and the particular way in which the researcher presented measures, worked hard to clarify and describe processes such as randomisation, and engaged with the participant. When talking about randomisation, 11 of the participants who displayed a sound understanding of the process discussed that they thought of this as the 'fairest' way.

'I thought that was quite fair to be honest, I mean there's no other way you can do it really.' (M001, male)

With regards to the specific measures, the participants experienced them as generally acceptable and three of the participants even identified some of the tasks as fun.
'Actually pretty fun though I mean cos the first one I did it right like the first time basically word for word that's pretty good knowing that my memory's that good.' (M009, male)

Some questions on the measures were perceived to be quite sensitive or deep (by eight of the participants), but participants felt they were still acceptable as they were aware they did not have to answer questions, and felt reassured by the researcher.

'Well some of them did make me a little uncomfortable you know she wanted to know more about things with my Dad and more about my past um but [unclear speech] uncomfortable but if they need to know it then they need to know it and I was just because a question makes me uncomfortable doesn't mean I'm not going to answer it you know what I mean so.' (M010, female)

'Yes no I did feel like I was able to say I don't want to answer she made it very clear at the beginning that I didn't have to answer them if I couldn't and so so yes I kind of relaxed about that.' (N002, female)

In addition to participants displaying understanding of the measures and processes, the overall presentation of the trial and researcher's general approach were deemed important.

'They were very friendly and they kind of, I don't think I would have done it had they not been as friendly as they were, if, if I had got there and they were just like very, you know, they weren't very friendly they weren't, I'm trying to think of the word that would describe them, well like, welcoming I'd probably thought, wwell, I don't like the idea of this, it's kind of $€$ and it wasn't like it was, even though it was run in with a hospital like, it's not anything like it, doesn't remind me of a hospital at all.' (M004, male)

'It's the way they said things which made me feel like I wanted to engage with the project.' (M005, female)

\section{Disclosure}

Closely linked to the theme of acceptability was the theme of disclosure and what facilitates this. Often ethics committees are concerned with how asking potentially difficult and sensitive questions may have an impact on vulnerable participants, who may feel torn between a sense of honesty and thoroughness, yet concerned with how their data will be treated, and possibly also concerned about judgements from the researcher, or any impact on their future care. Researchers may go a long way to allay such fears with proper training and full explanation of the study prior to informed consent, but the actual process of disclosure was discussed by participants as being a useful and therapeutic process in its own right. For example, some participants expressed surprise at the amount of disclosure. Here, a participant says of his baseline trial assessment:

'I talked about a lot, um, I admitted a lot more than I normally do to people.' (M006, male)

Most participants were surprised at their own natural level of disclosure. This was experienced as a positive surprise rather than as a negative worry, and might be seen as particularly beneficial for this participant group, who may not have had the opportunity to discuss these issues previously or report feelings being dismissed in the past. Ten of the participants also felt that these disclosures being a positive experience was as a result of the researcher being supportive, non-judgemental and empathetic.

'I think it was good to let people see into it, if you know what I mean, cos for so long I've kind of hidden away and not really told anyone about my problems, and not really opened up to anyone, so I think it now, it's nice to be actually, I need help with these things and it's ok to open up to them, so it was really good, and she was really nice as well which made it feel better.' (NOO2, female)

\section{Altruism}

Seven participants spoke at length of their keenness to be involved in research, for altruistic reasons. This was particularly apparent when considering the process of randomisation, as, despite potential ethical concerns about participants in the control group not receiving an intervention, there was an understanding and willingness to be involved as part of 'helping others'. 
'I know that's sort of a cliché thing to say, but it's true, I mean I'm not try'... to p, for sympathy, but I have had a terrible time, and I don't want other people to have it like, if you know, if I have children I wouldn't want them to have go through that I went through, and um, in generally I just, you know, want to take part in it for other people. (M006, male)

Altruism was particularly demonstrated when the participant had a good lay understanding of randomisation, as there was an awareness from those in the control group of participation 'for the greater good'.

'I suppose if the study is going to help figure out what'll help other people, then I don' see a problem with it, and it was a good op, whether I've been put in the right group or not, um, it's a good opportunity, yes, there's no harm in it, and it might help other people, so I don't see why not.' (M010, female)

'Ultimately that might help other people if we find that this therapy is good and helpful so I want to contribute in that sense.' (N010, male)

This demonstration of research participation being altruistically motivated provides some reassurance, from an ethical perspective, that randomisation in psychological studies is understood and specifically that participants in the control group do not feel unduly disadvantaged. However, it highlights the importance to continually guard against presuming an understanding of randomisation, as four participants demonstrated uncertainty, and this seemed to be linked to a feeling that in some way the baseline assessment may have had an impact on the trial randomisation process.

'Well I don't know, I'm not sure, I don't understand it now, so it could be random, or it would be, there's a decision been made, I can't tell.' (N006, male)

For these participants, confusion over randomisation appeared to arise from the detailed nature of the baseline assessment, conducted in such a way that allowed participants to feel able to open up to the researcher. Although this may be considered a positive, the perception following this may be that the outcome must have had some impact on group allocation, or mean they are already receiving the intervention.

'Yes I mean I did start to quiz all the questions and how detailed the assessment was, I did start to get the impression, well I mean surely I'm already in the therapy if al these questions are being asked of me, because why have they asked, why would all these questions be asked if I wasn't in the therapy? but now I've learned why they were all asked, because I'm going, you know, whether or not I'm going in the therapy I'd have been correlated with or compared, so now I understand.' (N010, male)

Despite some confusion, participants still saw the value of being part of the control group, highlighting the altruistic theme throughout.

\section{Engagement}

Considering the nature of the trial from which this qualitative study drew a sample, the issue of engagement was key. From a study design point of view there was concern that engagement in the study may be too demanding for participants, who were unwell and vulnerable, and had complex histories of social withdrawal characterised by a lack of engagement in ordinary daily activities. Encouragingly, however, seven participants spoke of engagement with the research processes, in addition to the actual study intervention. This engagement with the trial was experienced as a very positive step and potentially had additional benefits to the intervention itself.

'They [assessments] have been a turning point definitely it's just cos I think if they weren't there I'd have just kind of carried on like being full of shit, it's the only way I can describe it I suppose.' (M004, male)

A key consideration both practically and ethically, was the potential intrusiveness of the trial intervention. This was also a concern of service users from the service user involvement panel. Equally as there is potential to do good with the therapy intervention, there is also the potential to do harm. It was beyond the scope of these interviews to fully explore the therapeutic influence of the intervention but the initial feedback from the six participants in the intervention group was on the whole positive.

'Yes, um when me and (female therapist) will sit down and talk about things for a while, and then we'll hear a certain thing, um, and we'll write it down and go through feelings and emotions and cycles and stuff like that, and that I feel is a really good way to do it, because I never really thought of it that way, so you pull it apart and find out that actually I was feeling like that, and it was a situation where that's triggered it off, and stuff like that, so it's really, really helpful, um, and I'm going to try and start doing that like, on my own, she's given me some stuff to do that on my own as well, so.' (N002, female)

'I've got these things to fill in for next week, and I've got to think of different things I want, and it's just good because it's always, it's not like it's just for an hour a week, I'm concentrating on it, it's all week, I've got to be thinking about something and it's never leaving my mind.' (M009, male)

Although helpful, participants also described finding the intervention challenging, with three participants describing having to 'work on difficult things'.

'I hated it, but it wasn't because of (female therapist), it was just because I just don't like going out in crowded places, because I, you know, I just don't see it, so it was, it really, really surprised me at how much I hated it, and how scary it was to me, but um, it definitely helped a lot to notice things that I didn't notice before and also I just think it's, it's something I would do with, like, if I was going to counselling or something like that, it's not something that a counsellor would do.' (NOO2, female)

This is to be expected given the nature of the intervention. As pointed out by the participant, the intervention is quite different to other talking therapies ('counselling or something like that'). Exposing oneself in the presence of a therapist to feared situations is initially anxiety provoking and needs to be carried out sensitively and with a clear rationale. However, it is expected that, over time, anxiety reduces as the individual increases in confidence and learns they are able to cope in previously feared situations.

Despite the majority of those allocated to the therapy giving positive opinions about the SRCBT, one person expressed worries at the initial stages. This is understandable and expected, because of the personal nature of issues discussed within psychological intervention.

'A little apprehensive in the sense I think, just with counselling when you actually confront the problems, you sort of poking around inside, and you're digging things up that sometimes you might not want to confront, I think, and I think that aspect of it, just how counselling has hurt initially, that although most of it may be painful and hurt, but I think what I need to do is look at the greater image, the greater perspective as it were, and you know realise that, um, either what might be painful initially to go through, is probably for the better.' (N010, male)

\section{Discussion}

\section{Main findings}

The findings reported above can be summarised under the key thematic areas of practicalities, acceptability, disclosure, altruism and engagement. These themes highlight the potential benefits of participating in an RCT from a participant perspective. Although there are potential ethical issues of conducting research of this kind, the findings of this study suggest that when these are managed appropriately, involvement in research can have positive perceived effects for participants, beyond receiving the trial intervention.

\section{Comparison with existing research}

There is currently very little existing research addressing participants' experiences of the level of involvement required to partake in research and participant views of research processes. To our knowledge, there is no current research investigating the views of young people with high levels of social withdrawal in relation to involvement in research. Previous literature has not identified the practicalities required to make participation possible, and potentially even beneficial, for participants, especially particularly participants who are withdrawn or disabled. 
However, Byrne \& Morrison $^{6}$ investigated participant perspectives of monitoring assessments within an RCT evaluating early detection and prevention of psychosis. Themes of interpersonal engagement, informality and normalisation, and 'opening up' were identified, with participants highlighting the importance of an informal friendly manner that was also mirrored in the current findings around engagement and disclosure. Such a personcentred approach has also been highlighted as a valued attribute of effective engagement throughout qualitative research in early detection and intervention for psychosis (see, for example, O'Toole et al, Lester et al and Harris et al). ${ }^{14-16}$

Simmonds et al ${ }^{4}$ investigated patients' reasons for taking part and remaining in a trial assessing the effectiveness of CBT in addition to usual primary care for patients with treatmentresistant depression. Participants listed 'try anything to feel better', 'to help others with depression in the future', and 'the researchers were friendly' as the main reasons for taking part. This is consistent with the altruism theme identified in the current findings and the important qualities of the researcher subsumed within the themes of disclosure and engagement. Although motivation to help others is identified throughout literature around taking part in research trials, it is particularly notable that this is still applicable for this highly withdrawn client group.

The secondary aim of this study was to examine potential benefits of taking part in research $v$. ethical concerns and potential risks from participant perspectives. ${ }^{1,2}$ The acceptability of research methods and assessment measures found in this study has not been identified in previous literature from a participant perspective. The findings imply that it is the careful management of potential risks, rather than that the presence of risk per se, that should be of importance from an ethical perspective. For example, the risk of overburdening participants with potentially intrusive baseline assessments can be managed by ensuring that research assessments are sensitively and flexibly administered. The potential benefits of taking part in research are evident in the themes of acceptance, altruism and engagement reported in this paper. It was clear to us as researchers that participants reflected on their initial trial involvement in a positive and beneficial sense. This supports work with other at-risk populations. For example, qualitative work with those at risk of suicide has found involvement in research is more likely to be beneficial for participants than to invoke harm. ${ }^{17}$

\section{Implications}

This study provides learning points for future research in this area. The identification of the way in which assessment measures are presented and the interpersonal nature of the researcher as possibly being crucial to the acceptability of research procedures demonstrates the importance of thorough training of all those recruiting into a psychological intervention trial, to ensure consistency of understanding across study participants. The practicalities mentioned have costing implications in the design of further research projects with this client group because of the importance of flexibility in the timing and location of assessments outlined above.

Quality control and appraisal of recruitment and consent procedures must also be continually monitored. For some participants, confusion over randomisation appeared to arise from the detailed baseline assessment being conducted in such a way that allowed participants to feel able to open up to the researcher. Although this may be considered a positive, the perception following this may be that the outcome of the detailed assessment must have had some impact on the allocation to therapy, or mean they already are in therapy. There is a need to guard against complacency and ensure thorough explanation of randomisation for all trial participants. It may also be necessary to engage in a process of ongoing reiteration throughout the period of a trial of the importance of randomisation.

Furthermore, it is worth bearing in mind the findings of this study when designing future services for this client group. Although the study is small scale, it is important to consider the key approaches that make the participants feel valued and listened to, in order to design services that are effective. However, these links must be drawn tentatively as these early exploratory qualitative findings clearly require further investigation.

The findings of this study go some way to addressing some ethical concerns raised regarding psychological research of this type. For example, disclosure, although a concern, can also be beneficial and validating to participants, if managed and responded to appropriately. An additional concern that may be raised is the burden on participants of completing assessment tools. Again, although this needs to be managed flexibly, the findings of this study suggest that not only is completing assessments of this type acceptable but that participants may even gain something from this process, even if they are not randomised to the intervention condition. Further planned qualitative work with participants from both the intervention and control groups at trial follow-up will be reported at a later date to explore experiences of receiving the intervention compared with receiving TAU. It is likely that these future findings will assist in offering a detailed explanatory context for the trial findings and may further contextualise the findings reported here about participant involvement in research. Finally, this study also suggests that a vulnerable and hard-to-engage group can be successfully recruited into a study without adverse effects.

\section{Strengths and weaknesses}

The study was small and exploratory, and thus findings cannot be considered generalisable to other populations or to other research settings beyond this trial of SRCBT. Similarly, the convenience sampling approach, although practical, does not allow for wider conclusions to be drawn that may be applied to larger populations. In addition, participants may still have perceived these interviews to be part of the wider trial. In order to reduce this perception, participants were reassured that the interviewer (R.C.) was independent from the rest of the trial and negative feedback would be welcomed. Despite this, some participants may still have been reluctant to express more negative opinions.

However, the research findings have transferability within this population. ${ }^{18}$ Participant experiences explored in this paper are consistent with previous research projects that the research team has been involved in, ${ }^{5,6}$ and corroborate findings from the wider health services research literature of beneficial patient effects of research trial involvement (for example, Carroll et $a l^{3}$ ). Qualitative analysis for this study has been vigorously and systematically applied, and verified by independent members of the research team, in addition to feedback from a service user involvement panel. This is a particular strength of the method, maximising transferability of the findings.

\section{Funding}

This study was funded by NIHR Health Technology Assessment grant: 10/104/51 PRODIGY Trial: ISRCTN47998710 (UKCRN trial registration number: 13341). This article presents independent research funded by the National Institute for Health Research (NIHR). The views expressed are those of the authors and not necessarily those of the NHS, the NIHR or the Department of Health.

Caitlin Notley, BSC, PhD, UK Society for the Study of Addiction Research Fellow, Norwich Medical School, University of East Anglia, Norwich; Rose Christopher, BSC, 
Joanne Hodgekins, BSC, PhD, ClinPsyD, Department of Clinical Psychology, Norwich Medical School, University of East Anglia, Norwich; Rory Byrne, BSc, Greater Manchester West Mental Health NHS Foundation Trust, Psychosis Research Unit, Manchester; Paul French, BSc, PhD, ClinPsyD, Greater Manchester West Mental Health NHS Trust, Manchester and Department of Psychological Sciences, The University of Liverpool, Liverpool; David Fowler, MSC, CPsychol, Department of Psychology, University of Sussex, Brighton, UK

Correspondence: Caitlin Notley, Norwich Medical School, Norwich Research Park, University of East Anglia, Norwich, NR4 7TJ, UK. Email: c.notley@uea.ac.uk

First received 14 Feb 2014, final revision 4 Jul 2014, accepted 27 Jul 2014

\section{Acknowledgements}

We would like to thank all individuals who participated in the qualitative interviews described in this paper. Becky McIntyre assisted with qualitative interviewing in Manchester.

\section{References}

1 Ross S, Grant, A, Counsell, C, Gillespie, W, Russell, I, Prescott, R. Barriers to participation in randomised controlled trials: a systematic review. J Clin Epidemiol 1999; 52: 1143-56.

2 Haroun N DL, Haroun A, Cadenhead K, S. Risk and protection in prodromal schizophrenia: ethical implications for clinical practice and future research. Schizophr Bull 2006; 32: 166-78.

3 Carroll $R$, AntiquaJ, Taichman $D$, Palevsky $H$, Forfia $P$, Kawut $S$, et al. Motivations of patients with pulmonary arterial hypertension to participate in randomized clinical trials. Clin Trials 2012; 9: 348-57.

4 Simmonds B, Turner N, Thomas L, Campbell J, Lewis G, Wiles N, et al. Patients' experiences of participating in a large-scale trial of cognitive behavioural therapy for depression: a mixed methods study. Fam Pract 2013; 30: 705-11.

5 Morrison AP, Stewart SL, French P, Bentall RP, Birchwood M, Byrne R, et al Early detection and intervention evaluation for people at high-risk of psychosis-2 (EDIE-2): trial rationale, design and baseline characteristics. Early Interv Psychiatry 2011; 5: 24-32.
6 Byrne RE, Morrison AP. Young people at risk of psychosis: their subjective experiences of monitoring and cognitive behaviour therapy in the early detection and intervention evaluation 2 trial. Psychol Psychother 2014; 87 $357-71$

7 Fowler D, French P, Hodgekins J, Lower R, Turner R, Burton S, et al. CBT to address and prevent social disability in early and emerging psychosis. In CBT for Schizophrenia: Evidence Based Interventions and Future Directions (ed C Steel): 143-67. John Wiley \& Sons Ltd, 2013.

8 First M, Spitzer, R, Gibbon M, Williams, J. Structured Clinical Interview for DSM-IV-TR Axis I Disorders, Research Version, Patient Edition. (SCID-I/P). Biometrics Research, New York State Psychiatric Institute, 2002.

9 Yung AR, Phillips L, McGorry P, Ward J, Donovan K, Thompson, K. Comprehensive Assessment of At Risk Mental State (CAARMS). Department of Psychiatry, University of Melbourne, Melbourne, PACE Clinic, 2002.

10 Short S. Review of the UK 2000 Time Use Survey. Office for National Statistics, 2006

11 Robson C. Real World Research: A Resource for Users of Social Research Methods in Applied Settings. Sage Publications, 2011.

12 Notley C, Green G, Marsland L. Qualitative Analysis. In An Introduction to Health Services Research (ed D-M Walker): 327-42. Sage Publications, 2014.

13 Danermark B, Ekstrom M, Jakobsen L, Karlsson JC. Explaining Society Critical Realism in the Social Sciences. Routledge, 2002.

14 O'Toole MS, Ohlsen RI, Taylor TM, Purvis R, Walters J, Pilowsky LS. Treating first episode psychosis - the service users' perspective: a focus group evaluation. J Psychiatr Ment Health Nurs 2004; 11: 319-26.

15 Lester $\mathrm{H}$, Marshall $\mathrm{M}$, Jones $\mathrm{P}$, Fowler $\mathrm{D}$, Amos $\mathrm{T}$, Khan $\mathrm{N}$, et al. Views of young people in early intervention services for first-episode psychosis in England. Psychiatr Serv 2011; 62: 882-7.

16 Harris K, Collinson C, das Nair, R. Service-users' experiences of an early intervention in psychosis service: an interpretative phenomenological analysis. Psychol Psychother 2012; 85: 456-69.

17 Biddle L, Cooper J, Owen-Smith A, Klineberg E, Bennewith O, Hawton K, et al. Qualitative interviewing with vulnerable populations: individuals' experiences of participating in suicide and self-harm based research. J Affect Disord 2013; 145: $356-62$.

18 Malterud K. Qualitative research: standards, challenges, and guidelines. Lancet 2001; 358: 483-8. 